\title{
XXII. On the action of chlorine on cinnamic and benzoic acids
}

\author{
John Stenhouse Ph.D.
}

To cite this article: John Stenhouse Ph.D. (1845) XXII. On the action of chlorine on cinnamic and benzoic acids, Philosophical Magazine Series 3, 27:178, 129-137, DOI: 10.1080/14786444508645239

To link to this article: http://dx.doi.org/10.1080/14786444508645239

曲 Published online: 30 Apr 2009.

Submit your article to this journal

Џ Article views: 2

Q View related articles $\square$ 
through NO and the zenith. [Perhaps I may be allowed to suggest, that it would be an advantage to some of M. Gravier's results, were they reduced so as to have reference either to the pole of the earth, or to the magnetic pole as a zenith point.]

Through the medium of your valuble Journal, I desire to obtain for this communication the attention of men of science who are engaged in inferring from observations the general laws of phænomena. 'The advantage of the method I have proposed will be immediately seen by every person who will give it a trial; I shall not, therefore, occupy your space by any further statement of its superiority, but conclude with remarking, that unless the plan I have proposed be followed, the method of polar co-ordinates is inferior to that of rectangular co-ordinates, and is deprived of some of the advantages which it can be made to possess.

Cambridge, June 27, 1845.

XXII. On the Action of Chlorine on Cinnamic and Benzoic Acids. By John Stenhouse, Ph.D.*

I $N$ a short paper "On the Action of Peroxide of Lead on 1 Cinnamic Acid," published in vol. xix. of the Philosophical Magazine so long ago as the year 1841, I have incidentally mentioned that " when cinnamic acid is boiled with a solution of hypochlorite of lime, it is converted into benzoic acid with the formation of an oily liquid, which however is not oil of bitter almonds, as its taste and smell are quite different and much more aromatic." The extremely small quantity in which I at that time obtained this oily liquid unfortunately prevented me from being able to subject it to examination. As I was subsequently led to doubt if the acid into which the cinnamic acid had been decomposed was really the benzoic acid as I then imagined, I was induced, nearly six months ago, to resume the subject. While thus engaged, I was surprised to find the following passage in a paper on Chloranil, by Dr. Hofmann, read before the Chemical Society in December last: - "Neither phloridzine, phloretine, cumarine, nor cinnamic acid are transformable into chloranil. The first three furnish, by the action of chlorate of potash, yellow resinous bodies insoluble in water but soluble in alcohol, while the last is converted into a colourless oil, to which I shall recur in a future paper.$+ "$

From the perusal of the above extract I was immediately led to suspect that Dr. Hofmann had, by a different process,

* Communicated by the Author. † See Phil. Mag. S. 3. vol, xxvi. p. 205. Phil. Mag. S. 3. Vol. 27. No. 178. August 1845. K 
obtained the oil to which $I$ have already referred; and on repeating his experiment I was soon convinced that the oils produced by acting on cinnamic acid either with hypochlorite of lime or with chlorate of potash and muriatic acid are identical. I may mention that the cinnamic acid on which I operated was prepared from liquid storax by the following process:The storax was boiled for a couple of days with a very strong alkaline lye. The alkaline liquor when cold was diluted with a considerable quantity of water, which precipitated most of the resin which it beld in solution, and it was then decomposed by an excess of muriatic acid. The cinnamic acid precipitated in a very impure state, being mixed with a large quantity of resin.

If the alkaline liquor is neutralized while hot, this resinous matter melts and aggregates into large masses, which inclose much cinnamic acid, which is not easily separated from them, as they are not readily acted on by water. The cinnamic acid was purified by repeated crystallizations; the resin of the storax which had escaped decomposition during the first operation was again digested with an alkali, and the process above described repeated so long as it continued to yield any cinnamic acid. Cinnamic acid is usually prepared from balsam of Peru by means of an alcoholic solution of potash; balsam of Peru however is very difficult to decompose, does not yield quite so much cinnamic acid, and is more than twice the price of liquid storax, which is therefore by far the more ceconomical source of that acid.

\section{Action of Hypochlorite of Lime on Cinnamic Acid.}

When a quantity of cinnamic acid is dissolved in a saturated solution of hypochlorite of lime and subjected to distillation, a violent effervescence ensues, owing to the escape of carbonic acid gas, and a quantity of oil mixed with some acid and water passes over into the receiver. This oil is heavier than water, and has a very peculiar aromatic smell, intermediate between that of bitter almonds and Spirea ulmaria, though not exactly like either of them. Its taste is sharp and burning, pretty closely resembling that of cress seed. 'The oil was first washed with water to remove adhering acid. It was then left to stand for some days over a mixture of fused chloride of calcium and fragments of unslaked lime, to fiee it from moisture and muriatic acid; when poured off this mixture, the oil was strongly alkaline. It was then very cautiously distilled: the first small portion which came over was colourless and neutral, but what followed became more and more acid, the last portions exceedingly so, evolving fumes of muriatic acid and assuming a deep 
yellow colour. This operation was several times repeated with similar results. In this respect the oil very closely resembles chlorbenzine, which also cannot be distilled without being more or less decomposed. As the oil was always partially decomposed when distilled off fused chloride of calcium, another portion of it was repeatedly rectified with the vapour of water. The oil then came over quite colourless and neutral. This operation also freed it from a quantity of resinous matter which very readily forms in it. The purified oil when freed from water as well as possible was still more completely dried by being kept for some weeks over sulphuric acid in vacuo. When heated it readily catches fire and burns with a green-coloured flame, and emits fumes of muriatic acid gas. When heated with caustic potash, it is partially decomposed with the formation of chloride of potassium. When the oil is treated with strong nitric acid, it is readily attacked with copious evolution of deutoxide of nitrogen; and on the cooling of the liquid the oil is converted into a crystalline mass. The acid which is formed contains nitrogen and crystallizes readily; it is very soluble both in water and in alcohol; it forms soluble salts with the alkalies, and when neutralized it causes no precipitate in solutions of lime or silver salts. I have subjected portions of the chlorine oil, prepared at different times, to numerous analyses, from the results of whieh I am induced to believe that it is a carburetted hydrogen in which variable quantities of the hydrogen are replaced by chlorine. As the oil is a neutral body, I have been unable to determine its atomic weight, but $I$ expect that the examination of the acid which it forms with nitric acid will throw some light upon this point. I am at present occupied with this subject, the results of which I expect to communicate very soon. It has been already stated that the chlorine oil may also be readily procured by digesting cinnamic acid with chlorate of potash and muriatic acid. It is also invariably formed when a stream of chlorine gas is passed through a hot solution of cinnamic acid; so that there are three different methods by which it can be procured. The production of this oil forms an excellent test for the presence of cinnamic acid.

When either salicine or phloridzine are digested with a solution of hypochlorite of lime, carbonate of lime and resinous substances also united to lime are produced, but neither chloranil nor any oily or crystalline compounds.

Examination of the Acids formed by the Action of Hypochlorite of Lime on Cinnamic and Ben:oic Acids.

After the mixture of hypochlorite of lime and cinnamic acid $\mathrm{K} 2$ 
had ceased to yield oil to any considerable extent, it was transferred into a flask and boiled with a large additional quantity of a solution of bleaching powder, so as to ensure the complete decomposition of the cinnamic acid. The boiling was continued till every trace of the oil had been removed. A sediment slowly formed in the liquor consisting of carbonate of lime and resinous matter united to lime: the liquid was filtered in order to separate this precipitate: the clear liquid contained a soluble salt of lime united to an organic crystallizable acid. The lime-salt is exceedingly soluble, but does not crystallize, and forms, when concentrated, a shining transparent pellicle on the surface of the liquor. The salt was decomposed by an excess of muriatic acid, and digested till all the chlorine was driven off. As the liquor cooled, the acid precipitated in white voluminous Hocks; these were collected and washed with cold water to remove adhering muriatic acid. The crystals usually contain a little adhering resinous matter, from which they may be easily purified by repeated crystallizations. When the acid is heated in a quantity of water too small for its complete solution, it melts into an oily liquid, and in this respect differs from benzoic acid and resembles cinnamic acid: cold water dissolves very little of it, but it is tolerably soluble in boiling water, especially when the boiling is continued for some time. It is much less soluble than benzoic acid, however, and a good deal of it remains dissolved in the mother liquors. 'The acid dissolves very readily, both in alcohol and in æther. Water precipitates it from its alcoholic solution. When left to spontaneous evaporation, it readily crystallizes in small needles, which cross each other and form stars; they have a silky lustre. Its smell is disagreeable when it is impure, a little resembling that of naphthaline, but it diminishes as the acid grows purer, and at last wholly ceases: its taste is sharp and bitter, and affects the throat like benzoic acid. When gently heated, it melts and sublimes in crystals; when very highly heated, it catches fire and burns with a greenish flame, emitting much smoke. This led me to suspect that the acid contained chlorine, and on neutralizing a little of it with potash, treating the salt destructively, neutralizing it with nitric acid and testing it with nitrate of silver, I obtained a copious precipitate of chloride of silver. The acid itself produces no precipitate in a solution of nitrate of silver, but when it is neutralized by an alkali, a white flocculent precipitate appears. This precipitate is pretty soluble in boiling water, though much less so than benzoate of silver, but I was unable to obtain it in crystals. The acid gives no precipitate with acetate of lead, but a pretty dense precipitate 
with the subacetate. Several portions of the purified acid prepared at different times by treating cinnamic acid with hypochlorite of lime, were dried at $212^{\circ}$ and subjected to analysis.

(1.) 0.2011 grm. substance burned with chromate of lead gave 0.5085 carbonic acid and 0.0905 water.

Found.

Carbon . $68 \cdot 96$

Hydrogen 4.99

Oxygen - $\frac{26 \cdot 05}{100 \cdot 00}$

Almost pure benzoic acid.
Benzoic acid calculated.

14. Carbon. $1050 \quad 68 \cdot 85$

6 Hydrogen $75 \quad 4.91$

4. Oxygen $\frac{.400}{1525} \quad 26 \cdot 24$

(2.) a. 0.2455 gave $0.573 \mathrm{CO}_{2}$ and $0.095 \mathrm{HO}$.

b. 0.2332 gave $0.086 \mathrm{Ag} \mathrm{Cl}$.

Found.

Carbon . 63.65

Hydrogen 4.29

Chlorine. $9 \cdot 09$

Oxygen $\frac{.22 \cdot 97}{100 \cdot 00}$

Acid betwreen benzoic acid and the acid with one equivalent of chlorine.
Formula of acid with 1 equiv. of chlorine.

$$
\mathrm{C}_{14} \mathrm{Cl}_{4} \mathrm{O}_{3} \text {, HO. }
$$

14. Carbon . 1050*0 53.70

5 Hydrogen $62.5 \quad 3 \cdot 19$

1 Chlorine $442.6 \quad 22.63$

4. Oxygen. $\frac{400 \cdot 0}{1955 \cdot 1} \frac{20 \cdot 48}{100 \cdot 00}$

(3.) 0.2495 gave $0.471 \mathrm{CO}_{2}$ and $0.067 \mathrm{HO}$.

Found.

Carbon . 51 48

Hydrogen 2.98

$\mathrm{Cl}$ and $\mathrm{O} \quad 4.5 .54$

$\overline{100 \cdot 00}$

Acid with between one and two equivalents of chlorine.
Formula of acid with 2 equiv. of chlorine.

$$
\mathrm{C}_{14} \underset{\mathrm{Cl}_{2}}{\mathrm{H}_{3}} \mathrm{O}_{3} \text {, HO. }
$$

14. Carbon. 1050.0 44.02

4. Hydrogen $50.0 \quad 2.09$

2 Chlorine $885 \cdot 2 \quad 37 \cdot 01$

4. Oxygen - $400 \cdot 0 \quad \frac{16 \cdot 88}{100 \cdot 00}$

(4.) I. 0.3293 gave $0.5423 \mathrm{CO}_{2}$ and $0.0667 \mathrm{HO}$.

II. 0.2892 gave $0.477 \mathrm{CO}_{2}$ and $0.062 \mathrm{HO}$.

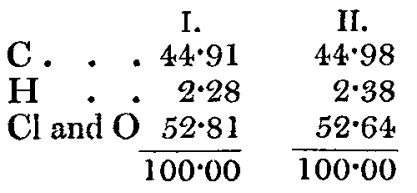

Corresponding to the acid with two equivalents of chlorine.
Formula of acid with two equiv. of chiorine.

Calculated.

14. C $1050 \cdot 0 \quad 4.4 \cdot 02$

4. $\mathrm{H} \quad 50 \cdot 0 \quad 2 \cdot 09$

$2 \mathrm{Cl} \quad 885 \cdot 2 \quad 37 \cdot 01$

4. O $400 \cdot 0 \quad 16.88$ 
(5.) a. 0.393 silver salt gave $0449 \mathrm{CO}^{2}$ and $0.064 \mathrm{HO}$. b. 0.5025 gave $0.268 \mathrm{Ag} \mathrm{Cl}$.

\begin{tabular}{|c|c|c|c|c|}
\hline & Found. & Formula of silver sa & $t$ with 1 a & chlonine. \\
\hline & $31 \cdot 16$ & 14 at. Carbon & $1050^{\circ}$ & 31.87 \\
\hline & $1 \cdot 80$ & 4 ... Hydroger & $50^{\circ}$ & $1 \cdot 31$ \\
\hline AgO & $43 \cdot 14$ & 1 ... Chlorine. & $442 \cdot 6$ & $13 \cdot 43$ \\
\hline & $23 \cdot 90$ & $3 \ldots$ Oxygen. & $300 \cdot 0$ & $9 \cdot 13$ \\
\hline & 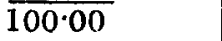 & $\mathrm{AgO}$ & $1451 \cdot 6$ & 44.06 \\
\hline rrespon & nding very nearly & & $3294 \cdot 2$ & $100 \cdot 00$ \\
\hline
\end{tabular}

with the silver salt with one equivalent of chlorine.

The first portion of the acid analysed was treated for a short time only with a comparatively small quantity of hypochlorite of lime; it resembled benzoic acid, and differed very considerably in its properties from the other portions of acid above described. In fact, as is evident from the results of its analysis, it was nearly pure benzoic acid, and contained scarcely a trace of chlorine, as $I$ ascertained by direct experiment. The $2 \mathrm{nd}, 3 \mathrm{rd}$, 4th and 5 th portions of acid had been repeatedly treated with large quantities of hypochlorite of lime and muriatic acid, some of them five and six times successively; and their analysis showed that their carbon, hydrogen and oxygen had diminished, and the amount of chlorine they contained increased in a corresponding proportion, thus forming a series of chlorinated acids in which one and two atoms of hydrogen are replaced by similar equivalents of chlorine. It is evident, as has been already observed, that the acid, the analysis of which stands first in order, is almost pure benzoic acid, the hydrogen of which has been scarcely at all attacked by the chlorine. The action of chlorine on cinnamic acid, therefore, is undoubtedly at first confined to converting it into the oily chlorine compound so often mentioned, and into benzoic acid; so that if its action could be interrupted exactly when this had been effected, these would be the only products. This precise period it is extremely difficult to hit, as the chlorine proceeds immediately to attack the benzoic acid and to replace one, two, and as we will. by and by see, so many as three equivalents of its hydrogen, thus forming a series of three distinct chlorinated acids. It is extremely difficult to obtain any one of these acids quite free from admixture, either of undecomposed benzoic acid on the one hand, or of more highly chlorinated acids on the other. The way in which this is most nearly accomplished, is by means of the silver salts, which are much less soluble than the benzoate of silver, and which consequently remains dissolved in their mother liquors when pretty dilute. The action of a mixture of chlorate of 
potash and muriatic acid on cinnamic acid is precisely similar to that of hypochlorite of lime, viz. the production of the oily compound, and subsequently of some of the series of chlorinated acids. As stated in a previous part of this paper, it is not necessary to have the chlorine in a nascent state, in order to effect the decomposition of the cinnamic acid. For if a stream of chlorine gas is sent through a hot solution of cinnamic acid, the chlorine oil and benzoic acid are immediately formed; and if the action of the chlorine is continued, the hydrogen of the benzoic acid is replaced just as in the cases already detailed.

There is a striking analogy, therefore, between the action of chlorine and of nitric acid upon cinnamic acid, the first effect of nitric acid upon cinnamic acid being confined to converting it into benzoic acid and oil of bitter almonds; but if the action of the nitric acid is pushed still further, the benzoic acid is also decomposed and converted into nitro-benzoic acid.

From what we have already stated, it was to be expected that in order to obtain these chlorinated acids, it would not be necessary to employ cinnamic acid, but that they would also be formed by acting directly upon benzoic acid itself, which is a much more easily procurable substance than cinnamic acid, and therefore a much more convenient source of these acids. This I found to be the case. Of course the benzoic acid gave no oil, but only the chlorinated acids.

The following are some of the results of chlorine on benzoic acid applied in the three several ways already described:-

A. Chlorobenzoic acids produced from benzoic acid by means of hypochlorite of line and muriatic acid.

I. a. 0.6092 substance burned with chromate of lead gave $0.990 \mathrm{CO}_{2}$ and $0.114 \mathrm{HO}$.

b. 0.4375 burned with bydrate of lime gave 0.6175 chloride of silver.

c. 0.3845 of the silver salt gave $0 \cdot 186$ chloride of silver.

d. 0.3425 of the silver salt gave 0.3525 carbonic acid and 0.034 water.

II. Another portion of benzoic acid treated still more frequently with hypochlorite of lime than the preceding.

0.34 .35 substance gave $0.509 .5 \mathrm{CO}_{2}$ and $0.610 \mathrm{HIO}$.

III. Acid which had been still longer treated than No. II. 0.3572 substance gave $0.510 \mathrm{CO}_{2}$ and $0.051 \mathrm{HO}$.

B. Chlorobenzoic acid obtained by acting on benzoic acid by a mixture of chlorate of potash and muriatic acid.

IV. 0.2066 substance gave $0.324 \mathrm{CO}_{2}$ and $0.036 \mathrm{HO}$. 
136 Action of Chlorine on Cinnamic and Benzoic Acids.

C. V. Acid obtained by means of chlorine gas sent through a hot solution of benzoic acid for about 56 hours.

a. 0.3334 substance, 0.6675 carbonic acid, and $0.097 \mathrm{HO}$.

b. 0.2068 substance, 0.4165 $\mathrm{CO}_{2}$, and $0.0665 \mathrm{HO}$.

c. 0.3522 substance gave with hydrate of lime 0.3125 chlo. ride of silver.

d. 0.8098 of the silver salt gave $0.9395 \mathrm{CO}_{2}$ and 0.1095 HO.

e. 0.414 silver salt gave 0.223 chloride of silver.

VI. Acid through a solution of which a stream of chlorine gas was passed for seventy hours.

a. 0.4158 substance gave $0.8053 \mathrm{CO}_{2}$ and $0.119 \mathrm{HO}$.

b. 0.336 of the silver salt gave 0.1775 chloride of silver.

'The following are the determinations per cent.

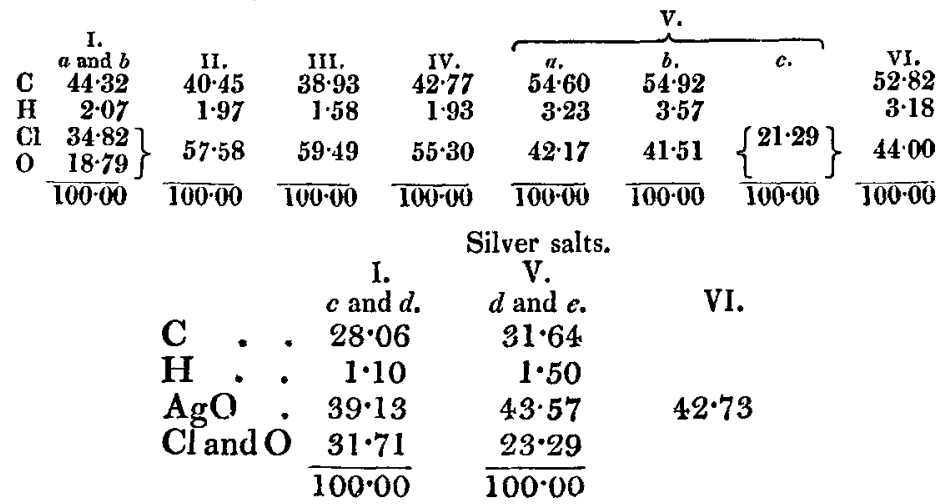

It will be observed by reference to the formulæ given in a previous part of the paper, that the acid A. obtained by hypochlorite of lime corresponds to the acid with two equivalents of chlorine. No. II., which was treated with still more hypochlorite of lime, gave a mixture containing acids with two and three equivalents of chlorine; and No. III., in which the treatment was carried still further, corresponds to an acid with nearly three equivalents of chlorine.

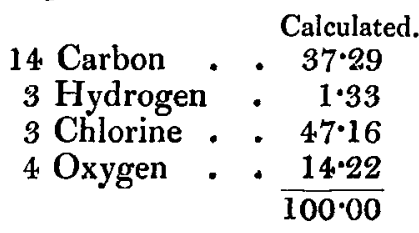

B. The acids obtained by means of chlorate of potash is a mixture of acids containing two and three equivalents of chlorine. 
C. marked V. is an acid containing one equivalent of chlorine; and the silver salt marked VI. is a mixture of acids containing one and two equivalents of chlorine.

Though several attempts were made, I did not succeed in obtaining any of the salts of these acids in distinct crystals. When boiled with a mixture of muriatic acid and alcohol, each of these acids formerl an æther, which in its smell and other properties closely resembled that of benzoic acid.

It is stated in most systems of chemistry, that chlorine has no action on solutions of benzoic acid, but from the facts already adduced, it is evident that this opinion is very incorrect. It would be unjust to conclude without referring to the experiments of $\mathrm{M}$. Herzog, detailed in Berzelius's Report for 1842 , p. 107 , Paris edition. M. Herzog says, "that when dry benzoic acid is exposed to the action of chlorine under the influence of solar light, the gas is absorbed and the acid is transformed into a reddish humid mass, having a disagreeable and fishy smell. When this is treated with carbonate of soda, it forms a brownish-red solution, while a resinous body having the smell of benzoin remains undissolved. When the alkaline solution is neutralized with nitric acid, a crystalline acid containing chlorine precipitates." The amount of the chlorine contained in it he did not determine. M. Hertzog also mentions that cinnamic acid is decomposed by chlorine in a similar way, yielding a chlorinated cinnamic acid. He makes no reference to the formation of any oil, nor does he appear to have attempted an analysis of any of these compounds whatever.

Glasgow, June 9, 1845.

XXIII. Proceedings of Learned Societies.

ROYAL SOCIETY.

Anniversary Meeting, Nov. 30, 1844.

r THE Marquis of Northampton in the Chair.

The noble President stated that the two Royal Medals had been adjudged by the Council to Mr. Boole, for his important papers on a General Method of Analysis*; to Dr. Andrews of Belfast, for his valuable paper on the Thermal Changes accompanying Basic Substitutions $f$; and the Copley Medal to Signor Matteucci of Pisa, for his discoveries in the magnetic electricity of animal nature.

The President then presented the Medals; after which he proceeded to state that the Society had lost some distinguished mem-

* An abstract of this paper will be found in vol. xxiv. p. 459.-ED.

+ Abstracts of Dr. Andrews's researches will be found in vol.xix. p. 183; vol. xxiv. p. 457 ; vol. xxv. p. 93, of this Journal.-ED. 